\title{
Bilateral double-layered patella in a patient with advanced knee osteoarthritis
}

\author{
P. Przybylski ${ }^{1}$, M. Skoczyński ${ }^{1}$, P. Tarkowski ${ }^{1}$, M. Tarczyńska², K. Gawęda² ${ }^{2}$ A. Drop ${ }^{1}$ \\ $11^{\text {st }}$ Department of Radiology, Medical University of Lublin, Poland \\ ${ }^{2}$ Department and Clinic of Orthopaedics and Traumatology, Medical University of Lublin, Poland
}

[Received: 6 May 2020; Accepted: 28 July 2020; Early publication date: 7 August 2020]

\begin{abstract}
Double layered patella (DLP) is a rare anomaly of the patella that may go undiagnosed, especially in patients with progressive knee dysfunction and early degenerative changes. Clinical symptoms such as motion-dependent pain and anterior knee pain most typically occur in adolescents or young adults; however, gradually increasing pain and early generalised degenerative changes have also been seen in patients over 40 years old.

Diagnosis of DLP could be difficult, especially in cases with coexisting arthrosis. $D L P$ is considered to be pathognomonic for the diagnosis of multiple epiphyseal dysplasia and usually coexists with other anomalies seen in this syndrome, such as hip dysplasia. In extremely rare cases, DLP can occur as a solitary disorder. The prevalence of such cases, however, is unknown, and they could be easily misdiagnosed. Computed tomography and magnetic resonance imaging are the most sensitive radiological methods used in DLP diagnosis. This case report presents a case of a bilateral DLP incidentally found in a 47-year-old patient with advanced arthritis referred for arthroplasty because of increasing symptoms of knee joint failure with no other abnormalities recorded. An important goal of our case study is to raise the awareness of this abnormality with radiologists and orthopaedic surgeons. (Folia Morphol 2021; 80, 3: 735-738)
\end{abstract}

Key words: bilateral double-layered patella, osteoarthritis

\section{INTRODUCTION}

Medical literature reports only a few cases of double layered patella (DLP). DLP is considered to be pathognomonic for the diagnosis of multiple epiphyseal dysplasia (MED) [7].

Early onset of clinical symptoms, such as increasing anterior knee pain and motion-dependent pain, are characteristic of young patients; however, dysfunctions as well as early, generalised degenerative changes have also been seen in patients over 40 years old with no history of trauma [10]. Because there are therapeutic implications to distinguishing DLP from other causes of knee failure in non-traumatic painful patients, imaging findings should be used to guide differential diagnosis.

This case report presents a case of an incidentally found bilateral DLP in a 47-year-old patient with advanced arthritis referred for arthroplasty because of increasing symptoms of knee joint failure with no other abnormalities recorded.

\section{CASE REPORT}

The patient (M.G.), aged 47, was referred from a county health centre to the Orthopaedic Clinic of the Medical University in Lublin, Poland, with in- 

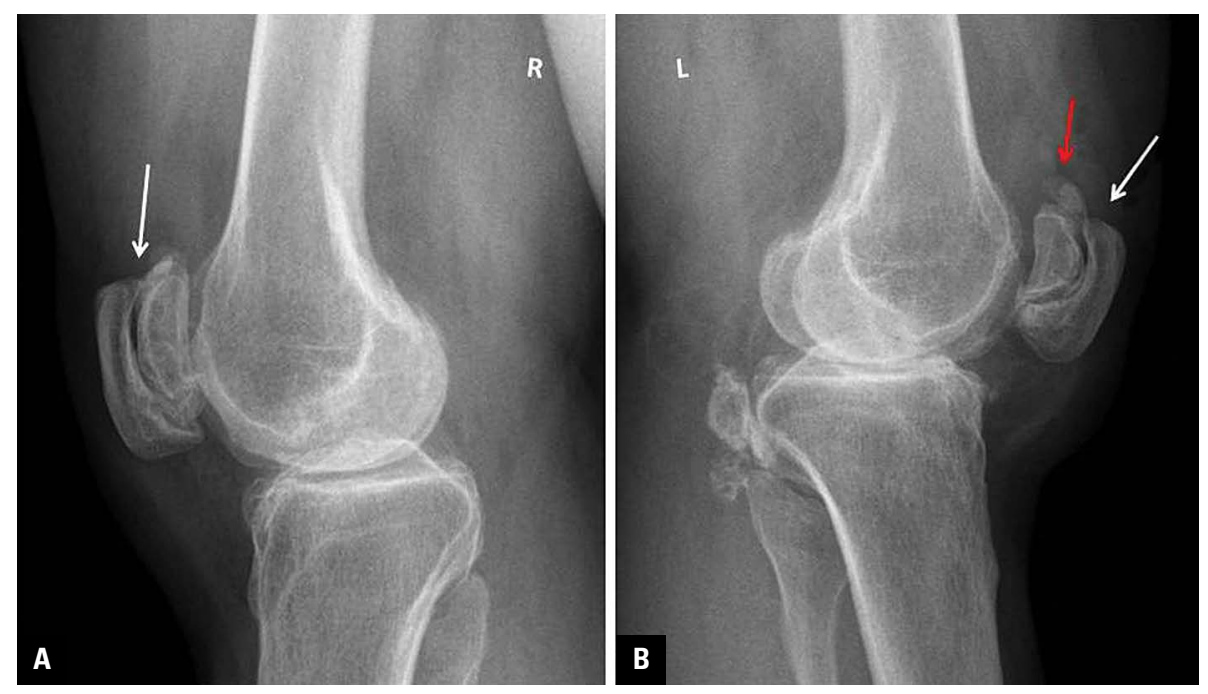

Figure 1. Lateral X-ray views of double-layered patella; A. Right patella (white arrow); B. Left (white arrow), additional separated segment in upper part of left patella (red arrow).

creasing symptoms of knee dysfunction and pain. On physical examination, the patient felt severe pain in both knees during movement and physical effort, especially the left knee, with a slight contracture in flexion and motor impairment of the left leg extension resulting in abnormal gait. The patient had not been diagnosed previously for these complaints. Physical examination also revealed a limited range of motion of 0 to 100 degrees of flexion for the right knee and 0 to 70 degrees for the left knee. The patient reported pain in both compartments in the area of the joint space. Antero-posterior and lateral view radiographs of both knees showed advanced arthritis, with more advanced lesions in the left knee. In lateral views two separated, parallel segments of both patellas were found (Fig. 1). The patient was referred for arthroplasty of the left knee. Computed tomography (CT) was performed a month later to assess bone structure. CT scans confirmed the presence of advanced degenerative changes in both knees and coexisting bilateral double-layered patella (Fig. 2). Additionally, several small segments were detected in the upper outer quadrant of the superficial surface of the left knee patella (Fig. 2B, D). During the arthroplasty, patellar segments were removed or fused. The articular surfaces of the distal femur and proximal tibia were replaced with prosthesis. Osteophytes that had formed on the patella and the edges of both bones were reduced. Following surgery, the patient underwent standard postoperative rehabilitation. Despite reminders, the patient never reported for follow-up.

\section{DISCUSSION AND CONCLUSIONS}

The patella, being the largest sesamoid bone in the human body, serves as an anatomic pulley (trochlea) for the quadriceps tendon. One of the most common patellar abnormalities is multipartite patella, which occurs in $0.2-6 \%$ of the population. A bipartite patella is the most prevalent form of this condition [12]. The partition is most often considered to be the consequence of a failed fusion of patellar ossification centres, but, other causes, such as trauma, tendon pulling on the patella, or insufficient vascular supply have also been proposed in the literature [11]. Available classifications of multipartite patella do not include a DLP [9]. DLP is currently considered to be pathognomonic for the diagnosis of MED, but single cases of this anomaly without coexistence of MED have also been described $[1,7]$. The dominant form of MED occurs in $0.01 \%$ of the population, but the prevalence of the recessive form is unknown [1]. It has been suggested that mutations in the COMP, DTDST, MATN3, COL9A1, COL9A2, and COL9A3 MED genes may be responsible for the occurrence of MED, while mutations in the DTDST and COL9A2 genes have been shown to coexist with the DLP phenotype $[1,5,10]$.

It is extremely difficult to estimate the prevalence of solitary DLP, because until now only a few cases have been reported in the literature available to the authors. First described by Buttner in 1925, DLP is an anomaly in which there are two patellar segments separated by a coronal septum: an anterior segment which is embedded in the quadriceps femoris tendon 

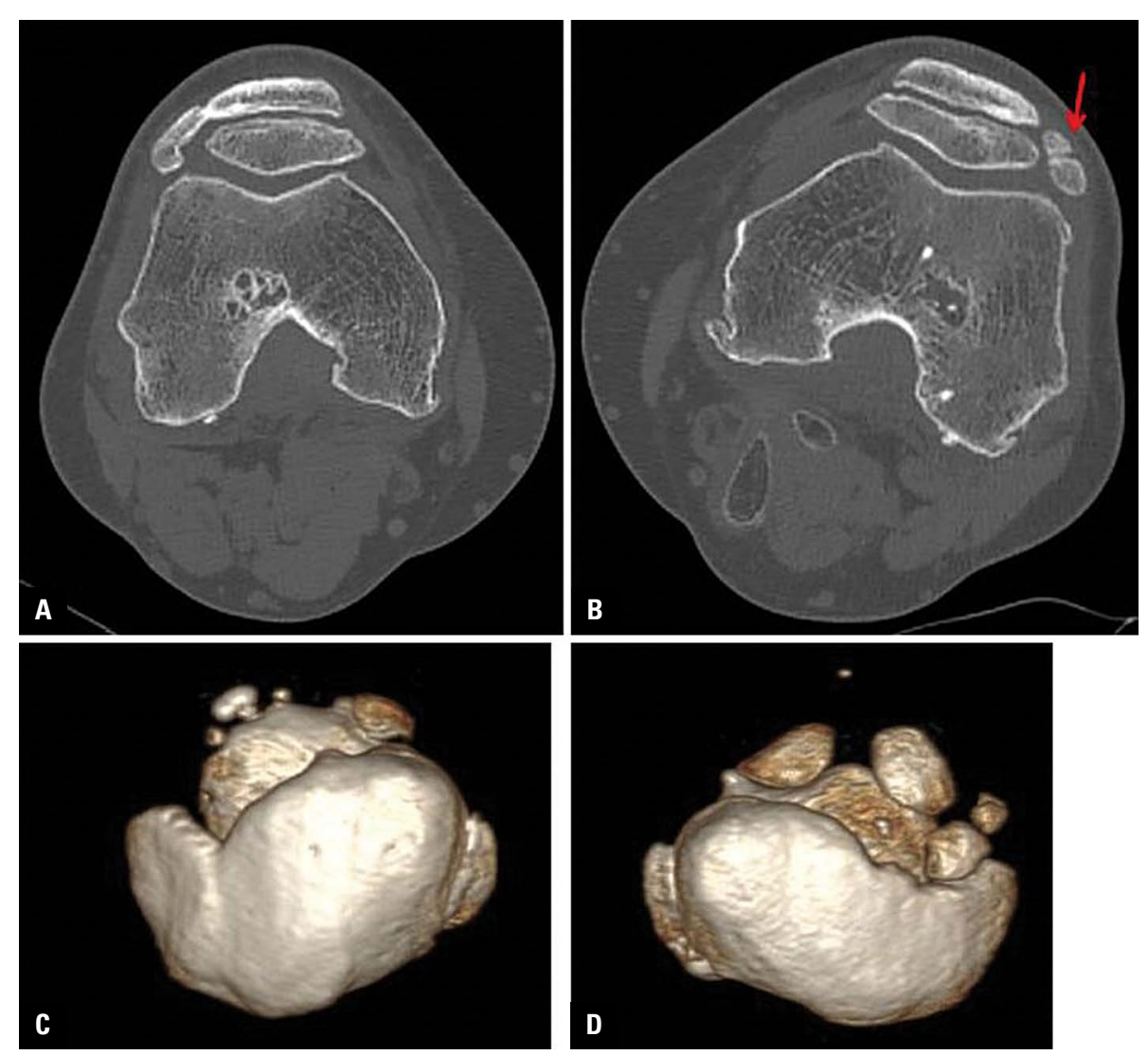

Figure 2. Computed tomography scans reconstructions; A. Axial view of right knee; B. Axial view of left knee, multiple segments in upper part of anterior layer are seen (red arrow); C. Three-dimensional reconstruction of right patella; D. Three-dimensional reconstruction of left patella.

and patellar tendons, and a posterior segment, which forms the articulating surface for the femur [7]. It is thought that DLP is bilateral in up to $40 \%$ of patients [3]. The interface between the two segments is cartilaginous $[4,7]$. Apart from complete DLPs, partial DLPs have also been described in the literature, often in association with fracture [5]. A partial DLP can also occur in patients without MED [3].

Double layered patella is sometimes asymptomatic, but it can also cause a variety of clinical problems. Clinical symptoms such as motion-dependent pain and anterior knee pain, clicking, locking or patellar dislocation most typically occur in adolescents or young adults [4, 7]; however, gradually increasing pain and early, generalised degenerative changes have also been seen in patients over 40 years old [10]. A delayed and painful movement of the posterior patellar segment that has no tendinous insertions, which causes painful snapping of the patella has also been reported [4]. DLP can be suspected based on clinical examination, especially in patients with a history of MED [7]. The diagnosis is confirmed following imaging (X-ray, CT or magnetic resonance imaging [MRI] scans) [8]. CT and MRI are the only modalities that enable precise morphological evaluation of both patellar segments. As a method that allows performing multiplanar and three-dimensional reconstructions, CT is an important preoperative tool. The layers can also be seen in ultrasound images, because the posterior segment is not entirely covered by the anterior segment.

Because DLP is a very rare abnormality, no standard treatment has been established so far. This means that therapeutic interventions are individually tailored to the patients' needs. Surgery has been performed in symptomatic patients both with and without a history of trauma. Resection of the posterior segment was proposed previously [2]; however, more recent work describes a good clinical outcome in patients who have undergone a surgery involving decortication of the separated bone segments and fixation using multiple stabilising elements $[4,5]$ or a single screw $[6]$. 
Patients who are referred for surgery may need a more extensive evaluation, including $\mathrm{CT}$ scanning, as CT scans can be used for planning treatment and printing three-dimensional models of the patella.

\section{Conflict of interest: None declared}

\section{REFERENCES}

1. Dahlqvist J, Orlén $H$, Matsson $H$, et al. Multiple epiphyseal dysplasia. Acta Orthop. 2009; 80(6): 711-715, doi: 10.3109/17453670903473032, indexed in Pubmed: 19995321.

2. Dahners LE, Francisco WD, Halleran WJ. Findings at arthrotomy in a case of double layered patellae associated with multiple epiphyseal dysplasia. J Pediatr Orthop. 1982; 2(1): 67-70, doi: 10.1097/01241398-198202010-00010, indexed in Pubmed: 7076836.

3. García-Mata S, Hidalgo-Ovejero A. Partial double-layered patella in a nondysplasic adolescent. J Pediatr Orthop B. 2016; 25(6): 561-563, doi: 10.1097/ BPB.0000000000000361, indexed in Pubmed: 27392305.

4. Goebel S, Steinert AF, Barthel T. Surgical management of a double-layered patella: a case report. Arch Orthop Trauma Surg. 2009; 129(8): 1071-1075, doi: 10.1007/ s00402-008-0701-z, indexed in Pubmed: 18677496.

5. Jørgensen A, Brandt E, Ulstrup A. Traumatic separation of osseous segments in a double-layered patella. Int J Surg Case Rep. 2017; 41: 352-355, doi: 10.1016/j. ijscr.2017.11.006, indexed in Pubmed: 29145110.
6. Leng HC, Foster P, Templeton PA. Fusion of double-layered patella using a single small fragment partially threaded screw: a case report. J Pediatr Orthop. 2011; 31(2): e9-e12, doi: 10.1097/BPO.0b013e318207454b, indexed in Pubmed: 21307701.

7. Milants A, De Maeseneer M, De Mey J. Double-layered patella (DLP) in multiple epiphyseal dysplasia (MED). J Belg Soc Radiol. 2017; 101(1): 8, doi: 10.5334/jbr-btr.1219, indexed in Pubmed: 30039000.

8. Nguyen MD, Everhart JS, May MM, et al. Bilateral double-layered patella: MRI findings and fusion with multiple headless screws: a case report. JBJS Case Connect. 2013; 3(2): e50, doi: 10.2106/JBJS.CC.L.00314, indexed in Pubmed: 29252402.

9. Oohashi Y, Koshino T, Oohashi Y. Clinical features and classification of bipartite or tripartite patella. Knee Surg Sports Traumatol Arthrosc. 2010; 18(11): 1465-1469, doi: 10.1007/s00167-010-1047-y, indexed in Pubmed: 20111951.

10. Sayilir S, Ekiz T. Early osteoarthritis and double-layered patella in a patient with multiple epiphyseal dysplasia. Arch Rheumatol. 2017; 32(3): 260-263, doi: 10.5606/ArchRheumatol.2018.6415, indexed in Pubmed: 30375546.

11. Smillie IS. Injuries of extensor apparatus. In: Injuries of the Knee Joint. 3rd Ed. Williams \& Wilkins, Baltimore, MD 1962: 272-277.

12. Weckström M, Parviainen $M$, Pihlajamäki HK. Excision of painful bipartite patella: good long-term outcome in young adults. Clin Orthop Relat Res. 2008; 466(11): 2848-2855, doi: 10.1007/s11999-008-0367-4, indexed in Pubmed: 18607662. 